\title{
Virtual reality reduces COVID-19 vaccine hesitancy in the wild
}

Clara Vandeweerdt ${ }^{1}$, Tiffany Luong ${ }^{2}$, Michael Atchapero $^{1}$, Aske Mottelson ${ }^{1}$, Christian Holz ${ }^{2}$, Guido Makransky ${ }^{1}$, and Robert Böhm ${ }^{1,3,4}$

${ }^{1}$ Department of Psychology, University of Copenhagen, Copenhagen, Denmark

${ }^{2}$ Department of Computer Science, ETH Zürich, Zürich, Switzerland

${ }^{3}$ Department of Economics, University of Copenhagen, Copenhagen, Denmark

${ }^{4}$ Copenhagen Center for Social Data Science (SODAS), University of Copenhagen,

Copenhagen, Denmark

August 2021 


\begin{abstract}

\section{Background}

Vaccine hesitancy poses one of the biggest threats to global health. Informing people about the collective benefit of vaccination due to community immunity has great potential in increasing vaccination intentions. Novel communication formats are needed to increase people's interest in and engagement with such information, boosting the intervention's effectiveness. This research investigates the potential for virtual reality (VR) to strengthen participants' understanding of community immunity, and therefore, their intention to get vaccinated.
\end{abstract}

\title{
Methods and Findings
}

In this pre-registered lab-in-the-field intervention study, participants $(n=222)$ were recruited in a public park. They either experienced the collective benefit of community immunity in a gamified immersive virtual reality environment ( $2 / 3$ of sample), or received the same information via text and images ( $1 / 3$ of sample). Before and after the intervention, participants indicated their intention to take up a hypothetical vaccine for a new COVID-19 strain (0-100 scale) and belief in vaccination as a collective responsibility (1-7 scale).

After the VR treatment, for participants with imperfect vaccination intention, intention increases by 9.3 points $(95 \% \mathrm{CI}$ : 7.0 to $11.5, p<0.001$ ). The text-and-image treatment increases vaccination intention by 3.3 points (difference in effects: $5.8,95 \%$ CI: 2.0 to $9.5, p=0.003$ ). The VR treatment also increases collective responsibility by 0.82 points (95\% CI: 0.37 to $1.27, p<0.001)$. A key limitation of the study is that it measures vaccination attitudes, but not behavior.

\section{Conclusions}

VR is an effective tool for increasing vaccination intention, more so than text and images, by eliciting collective responsibility. The results suggest that VR interventions can be applied "in the wild" and may thus provide a complementary method for vaccine advocacy. 


\section{Introduction}

Vaccination against most infectious diseases is an individual decision with positive externalities. That is, when individuals get vaccinated, they not only protect themselves, but typically also limit the probability that they will transmit the disease to others ${ }^{10}$. As such, even unvaccinated citizens can be indirectly protected from infection, known as community immunity or herd immunity ${ }^{10}$. With regard to the COVID-19 pandemic, it has been estimated that $60-90 \%$ of the population need to be vaccinated (depending, for instance, on the vaccine efficacy) to stop the spread of SARS-CoV-2 and, thus, to end the pandemic ${ }^{2,13}$.

Fully informed vaccination decisions require that people know and understand the individual costs and benefits of a vaccination, as well as its collective benefit. In line with the assumption that people care not only about their own but also about others' welfare, informing them about community immunity has sometimes (e.g., Pfattheicher et al. ${ }^{23}$ ) but not always (e.g., Hendrix et al. ${ }^{15}$ ) been shown to increase vaccination intentions (for a review, see Hakim et al. ${ }^{14}$ ). Interactive simulations have been particularly effective in increasing vaccine intentions $5,7,25$, potentially because they are more engaging $^{1}$ and, therefore, increase people's learning motivation ${ }^{3,7}$.

Building on these findings, in this study we investigate whether vaccination intention is increased by a gamified immersive VR experience showing how community immunity works. VR is a promising medium for health communication (cf. Freeman et al. ${ }^{11}$ ) because users find it both enjoyable and engaging as a learning tool ${ }^{17,26}$. Still, it has only just started to be tested as a tool for vaccine advocacy, showing some promising results but no significant effect on vaccination intentions ${ }^{20}$.

In our VR simulation, participants must either try not to infect other non-player characters in a virtual scene, or try not to get infected by them. All participants play two scenarios — starting with an environment in which few characters are vaccinated, followed by an environment where many characters are vaccinated. The simulation thus allows participants to experience community immunity from a first-person perspective, learning how much more slowly infection spreads when vaccination rates are high versus low. Moreover, by using gamification in an immersive VR simulation, participants are likely to be motivated and engaged with the learning content ${ }^{4,18}$.

\section{Method}

The design and analysis plan of this randomized control trial was preregistered on June 3rd, 2021, prior to accessing any data. Unless otherwise noted, all steps below follow the pre-registration plan (see here for the anonmyized plan). The full study procedure was approved by the relevant Institutional Review Board.

\section{Recruitment and procedure}

Participants $(n=222)$ were 207 passersby recruited in a public park in Copenhagen during the first weekend in June 2021, plus 15 passersby recruited one week earlier on campus at the University of Copenhagen. Respondents (39\% female, age range $=18-63$ ) participated in exchange for drinks and snacks. Table 1 contains key descriptive statistics.

After filling out an informed consent form and pre-treatment questionnaire, $2 / 3$ of the participants were randomly assigned to the VR treatment. The other $1 / 3$ were randomly assigned to read a text and see images explaining community immunity. All participants then filled out a post-treatment questionnaire. Finally, all participants also received the treatment they had not been assigned to initially (VR or text-and-image), and filled out a second post-treatment questionnaire. Figure 1 shows the trial profile.

\section{Treatments}

Below, we detail the content of both treatments: the VR simulation and the text-and-image treatment. 
Table 1: Characteristics of the study sample. Continuous variables are summarized as mean (standard deviation). Last two rows are \% of respondents who had maximum values on the outcome measures before receiving any treatment.

\begin{tabular}{ll}
\hline Sample characteristic & Result \\
\hline Age & $29.0(9.1)$ \\
$\%$ Female & $39 \%$ \\
\% Vaccinated & $16 \%$ \\
Previous VR experience (median) & $1-3$ times \\
Pre-treatment vaccination intention & $65.8(25.6)$ \\
Pre-treatment collective responsibility & $6.0(1.5)$ \\
\% Max pre-treatment vaccination intention & $12 \%$ \\
\% Max pre-treatment collective responsibility & $53 \%$ \\
\hline
\end{tabular}

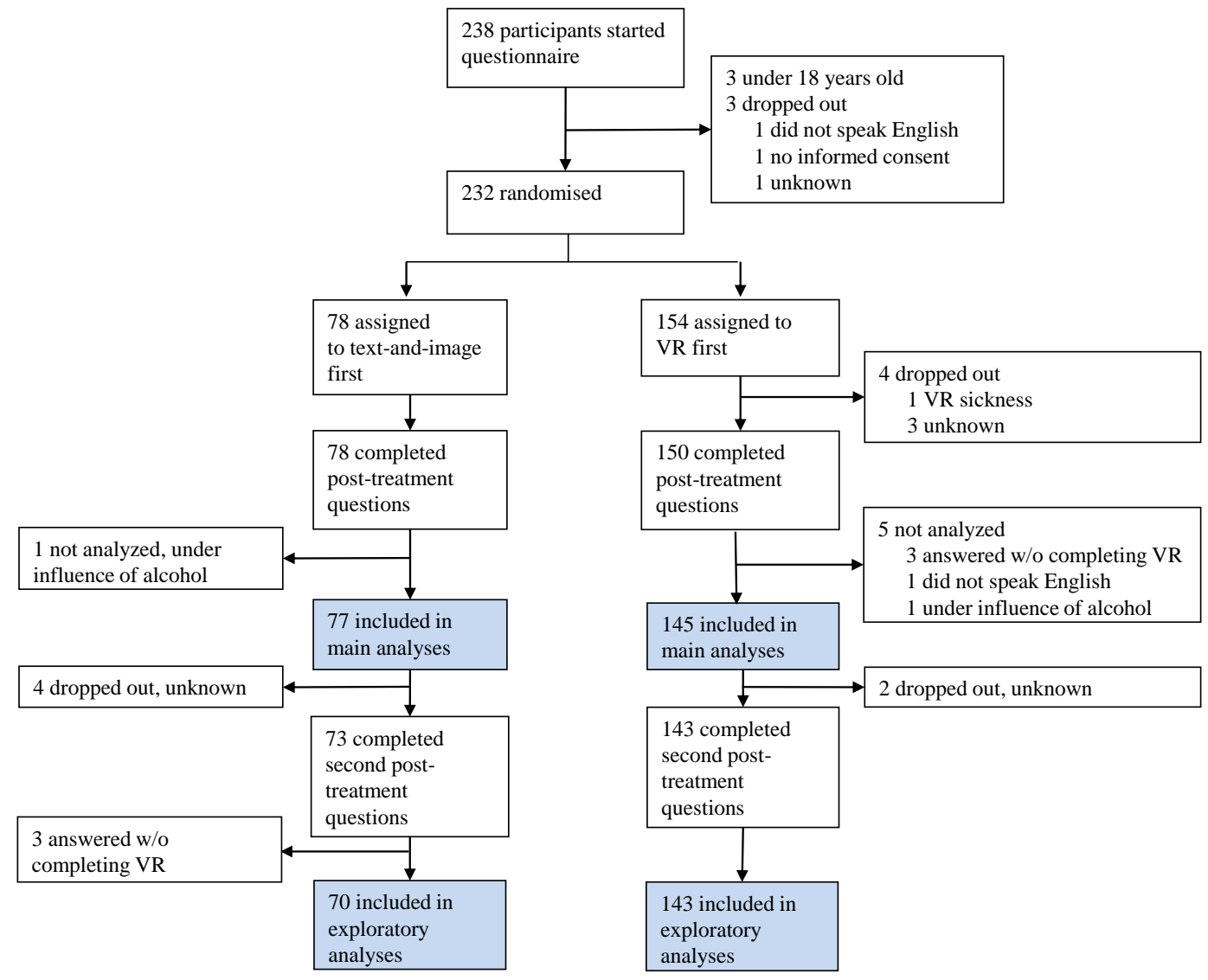

Figure 1: Trial profile showing flow of participants into treatment arms and analyses. Because participants largely self-administered the questionnaires and treatments, needing assistance only to start up the VR simulation, dropout reasons are sometimes unknown. 
VR. In the VR treatment, participants wore an Oculus Quest headset for a 5-10 minute simulation developed at SIPLAB (ETH Zurich). They were embodied as an older character matching their gender. They were told that their character is vulnerable to COVID-19.

Participants were randomly assigned to one of two versions of the VR simulation. In the avoid spreading version, the player character ("avatar") is already infected and the player must try not to infect others. In the avoid infecting version, the player character is uninfected and the player must try not to be exposed to infected characters.

In a first step, a tutorial showed the mechanics of the game. There would be healthy-andunvaccinated, infected (red clothing) and healthy-and-vaccinated (blue clothing) characters in the environment. Infected characters would spread the disease when coming too close to a healthy character. In a second step, participants were tasked with crossing a busy square to reach a marked destination, while avoiding contact with the other 130 characters in the square. In the first scenario, they did so in an environment where $20 \%$ of the virtual characters were vaccinated. In the second scenario, they crossed the busy square again, but with $70 \%$ of the characters being vaccinated.

When participants came into close contact with a character (infecting them or being exposed to their infection), they were made aware through graphics and haptic feedback (vibrating controllers). ${ }^{a}$ A small graph also helped them see how the disease spread between characters in the square, increasing the count of infected characters as they moved through the scenario. Figure 2 shows the square scene and spreading graph.

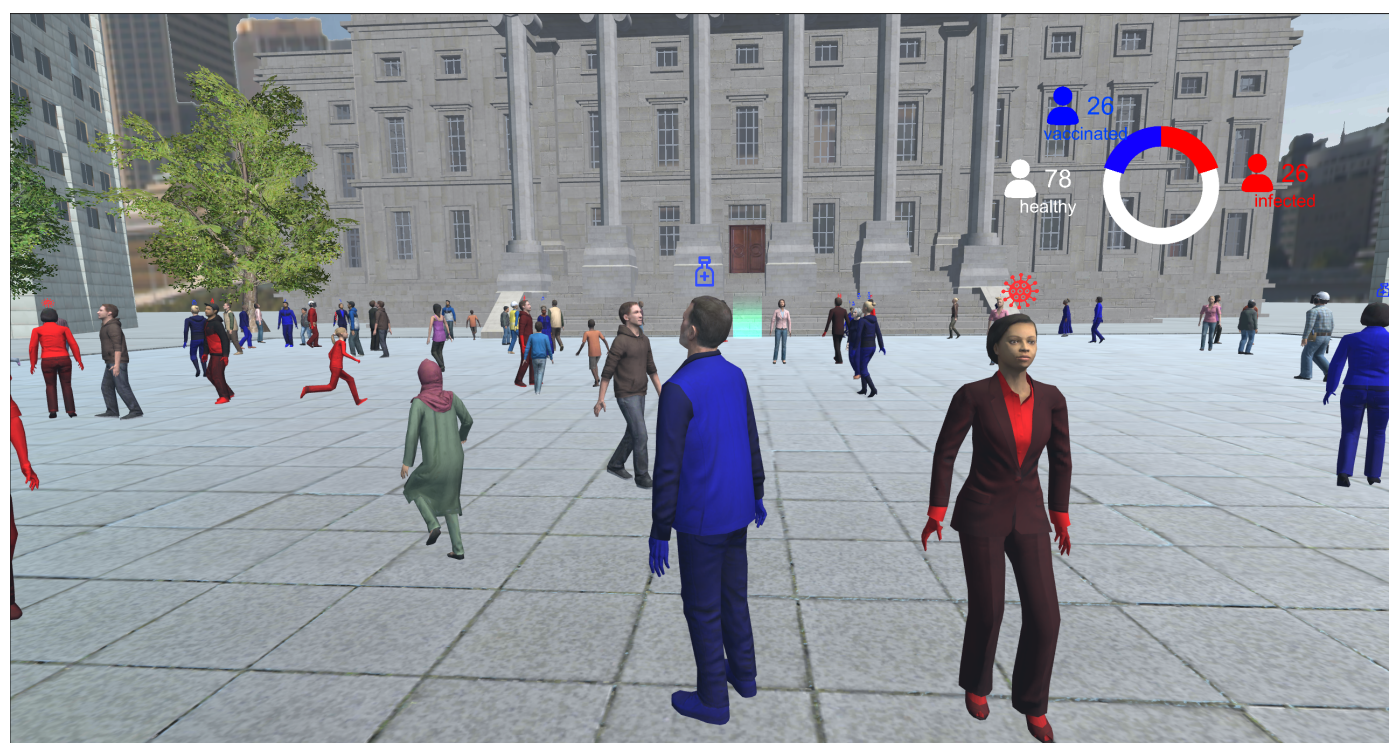

Figure 2: The busy square scene in VR, with feedback graph showing the number of infected, healthy and vaccinated characters.

Text-and-image. The alternative treatment, using text and images, displayed the definition of community immunity by the US Centers for Disease Control and Prevention, followed by two pictures (adapted from Betsch et al. ${ }^{7}$ ) with captions. The pictures represent communities where few or many people are vaccinated. Captions explained that in the low-vaccination community, many healthy but unvaccinated people are at risk of infection. In the high-vaccination community, few are at risk.

Both the VR and text-and-image treatment ended with a brief summary, highlighting the takeaway message ("As you can see, when many people are vaccinated the virus does not spread as fast and it creates a world that is safer for everyone. You can see the difference in [...] the low and high

\footnotetext{
${ }^{a}$ Close contact was defined as a $2 \mathrm{~m}$ radius around the character.
} 
vaccination scenarios"). A more detailed description of the VR treatment, including video, and the full text-and-image treatment, can be found in the study repository.

\section{Randomization and masking}

Randomization between treatment orderings happened within the Qualtrics survey software. Random assignment to a version of the VR simulation (avoid spreading or avoid infection) was tied to participants' ID numbers (even or uneven), which were allocated consecutively. Experimenters only assisted participants in starting up the VR simulation. They were blind to both the treatment ordering and the VR simulation version that each participant was assigned to.

\section{Outcome measures and hypotheses}

Two key measurements were taken before and after participants' first treatment, as well as after their second treatment: First, vaccination intention for a hypothetical new COVID-19 strain was assessed (0-100 scale; adapted from ${ }^{7}$ ). This measure was pretested in a pilot study available in the supplemental material. Second, seeing COVID-19 vaccination as a collective responsibility was assessed (1-7 scale; $\left.{ }^{12}\right)$. The supplemental material details the wording of these two items, and all other measures collected in the study.

\section{Hypotheses}

All preregistered hypotheses are about the effect of the participants' first treatment on the two outcome measures, either the VR treatment or the text-and-image treatment. We devised the following hypotheses:

H1. Vaccination intention increases after the VR treatment.

$\mathrm{H} 2$. Vaccination intention increases more after the VR treatment than after the text-and-image treatment.

H3. Collective responsibility increases after the VR treatment.

H4. Collective responsibility increases more after the VR than than after the text-and-image treatment.

\section{Results}

Figure 3 (left panel) illustrates the effect of each treatment on vaccination intention as the difference between measurements before and after the first treatment. The supplemental material presents the full distribution of individual treatment effects.

Comparing vaccination intention between the pre-treatment and first post-treatment measure, for participants who did not already have maximally positive vaccination intention $(n=195)$, we find that the VR treatment increased vaccination intention by 9.3 points $(95 \% \mathrm{CI}: 7.0$ to $11.5, p<0.001)$. The VR treatment is more effective than the text-and-image treatment, which only increases vaccination intention by 3.3 points (difference in effects: $5.8,95 \%$ CI: 2.0 to $9.5, p=0.003$ ).

Comparing collective responsibility pre- and post-treatment, for participants who did not already score the maximum on collective responsibility $(n=104)$, we find that the VR treatment increases collective responsibility by 0.82 points ( $95 \% \mathrm{CI}: 0.37$ to $1.27, p<0.001)$. The VR treatment is once again more effective than a text-and-image treatment, which increases collective responsibility by just 0.43 points, though the difference is not significant (difference in effects: $0.39,95 \% \mathrm{CI}: 0.36$ to $1.14, p=0.275$ ). Yet, note that the power to detect significant treatment differences is lower here, due to the smaller number of "moveable" participants with less-than-perfect perceptions of collective responsibility. 

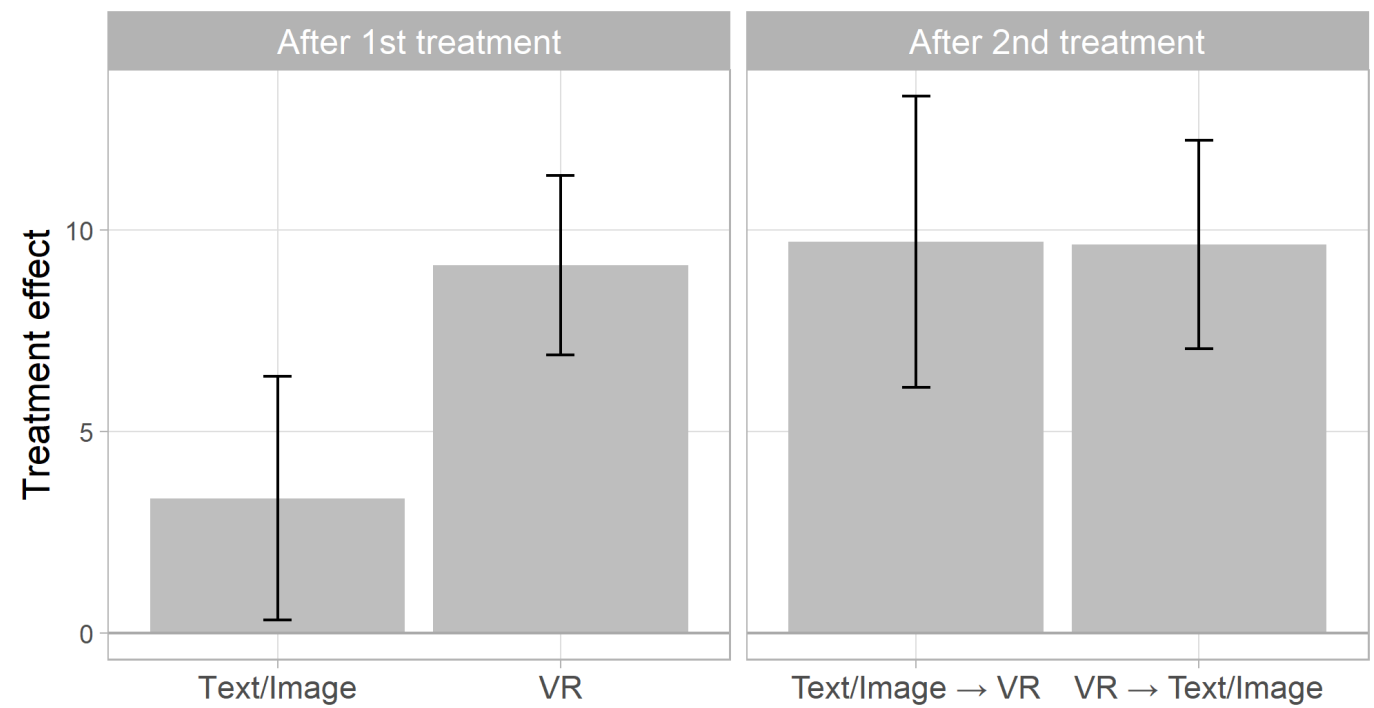

Figure 3: Change in vaccination intention from pre-treatment measurement, after first treatment $(n$ $=195$, left panel $)$ and after both treatments $(n=189$, right panel), leaving out participants with maximum pre-treatment vaccination intention. Error bars are $95 \%$ CIs

Further, we conducted an exploratory analysis on whether the VR treatment further increases vaccination intentions after a text-and-image treatment. Indeed, as shown in the right panel of Figure 3, we find that for participants who experienced the text-and-image treatment first and did not have maximum pre-treatment vaccination intention, the subsequent VR treatment further increased vaccination intention by 6.3 points $(95 \% \mathrm{CI}: 4.2$ to $8.3, p<0.001)$. In contrast, for those who received the text-and-image treatment after the VR treatment, there was no significant further increase in vaccination intention (effect: $0.7,95 \% \mathrm{CI}$ : -0.7 to $2.2, p=0.309$ ).

We also explored any potential difference between the effectiveness of the two versions of the VR treatment: the one where participants avoided spreading COVID-19 and the one where they avoided infection with COVID-19. There is no difference in the effect of these two versions on either vaccination intention (difference in effects: $0.2,95 \% \mathrm{CI}:-4.2$ to $47, p=0.912$ ) or collective responsibility (difference in effects: $-0.003,95 \% \mathrm{CI}:-0.46$ to $0.45, p=0.989$ ).

Finally, we asked all participants who completed the full study $(n=208)$ whether learning about community immunity via VR and text/pictures was fun, and whether they would like to receive more health communications via VR and text/pictures (1-5 scale). Compared to their ratings of text, participants rated VR as more fun (difference in means: $0.23,95 \% \mathrm{CI}: 0.11$ to $0.35, p<0.001$ ). There was no difference on how much participants wanted to receive future health communications via the two media (difference in means: $-0.06,95 \% \mathrm{CI}$ : -0.18 to $0.05, p=0.301$ ).

\section{Discussion}

We provide seminal evidence that a first-person experience of vaccinations' collective benefits in immersive VR can increase vaccination intentions. Further, the VR treatment is nearly three times more effective than communicating the same content via text and images. As the intended effect of both treatments is quite clear, the VR treatment's greater effectiveness shows that its impact cannot be reduced to demand characteristics. This is further supported by the finding that adding the VR treatment after the text-and-image treatment further increased vaccination intention, whereas adding the text-and-image treatment after the VR treatment did not provide any further benefit.

Our results suggest that a VR intervention communicating about the collective benefit of vaccination clearly goes beyond merely providing information. The results fit into a growing literature 
on the effectiveness of communicating about community immunity on vaccination intentions (see Hakim et al. ${ }^{14}$ for a review) and provides a solution to the problem that such interventions appear more effective when they are more engaging, such as via interactive simulations ${ }^{5,7,25}$, and when they elicit emotions, such as empathy ${ }^{23}$. As we demonstrate here, immersive VR is an effective alternative communication medium that can convey the collective benefit of vaccination in a highly engaging and emotional way.

There are several potential mechanisms for why the VR treatment leads to changes in behavioral intentions that deserve further investigation in future research. Our participants reported greater fun with the VR treatment than with the text-and-image treatment. Previous research has shown that immersive VR increases participants' interest in the content domain ${ }^{22}$ and enjoyment ${ }^{21}$, which can increase attention and effort ${ }^{18}$ to understand a topic. Immersive simulations also induce a sense of presence ${ }^{24}$, which can cause participants to associate negative and positive emotions with low and high vaccination rates, respectively. Such effects are likely to further increase when elements of gamification are used ${ }^{4}$, as in the present study. Taken together, there are both cognitive and emotional features of the VR treatment that are likely influence behavioral intentions.

The current research has some limitations. Firstly, our outcome measures were vaccination intention and collective responsibility. We used established measures for these constructs and both have been linked to self-reported vaccine uptake ${ }^{8,19}$. Nevertheless, future research should investigate the effects on actual vaccination behaviour. Secondly, our sample was composed of passersby in a public park, hence, people who had greater interest in VR may have been more likely to participate. Yet, showing the effectiveness of VR in increasing vaccination intentions "in the wild" indicates the great potential of this technology for vaccine advocacy, particularly when targeting younger people. Thirdly, although we found a substantial change in vaccination intentions, future research could develop an even more effective VR treatment. For example, future versions may improve the experience's narrative, or create more empathy with a main character who is especially vulnerable to the target disease. Finally, although the present study focused on COVID-19 vaccination intentions, the VR treatment can also easily be adapted to, and tested for, different infectious diseases.

Our research contributes to a potential paradigm shift in health communication generally, and vaccine advocacy in particular. Finding novel methods to reduce vaccine hesitancy is of utmost importance $^{6,9,16}$. VR provides a flexible tool to create more engaging and interactive learning experiences. For vaccine and community immunity information in particular, where it is crucial to reach and engage healthy members of the population (including young adults), VR has strong potential to complement more traditional communication channels and, therefore, contribute to decreasing the threat from infectious diseases.

\section{Data sharing statement}

Anonymous individual participant data, plus analysis files a data dictionary with variable descriptions, are available to anyone from the study repository. The repository also includes the study protocol, pre-analysis plan and informed consent form.

\section{Declaration of interests}

The authors know of no actual or potential conflict of interest related to this study.

\section{References}

1 Allcoat D, von Mühlenen A. Learning in virtual reality: Effects on performance, emotion and engagement. Research in Learning Technology 2018;26.

2 Anderson RM, Vegvari C, Truscott J, Collyer BS. Challenges in creating herd immunity to SARS-CoV-2 infection by mass vaccination. The Lancet 2020;396(10263):1614-1616. 
3 Annetta L, Mangrum J, Holmes S, Collazo K, Cheng MT. Bridging realty to virtual reality: Investigating gender effect and student engagement on learning through video game play in an elementary school classroom. International Journal of Science Education 2009;31(8):10911113.

4 Bai S, Hew KF, Huang B. Does gamification improve student learning outcome? Evidence from a meta-analysis and synthesis of qualitative data in educational contexts. Educational Research Review 2020;30:100322.

5 Betsch C, Böhm R. Moral values do not affect prosocial vaccination. Nature human behaviour 2018;2(12):881-882.

6 Betsch C, Böhm R, Chapman GB. Using behavioral insights to increase vaccination policy effectiveness. Policy Insights from the Behavioral and Brain Sciences 2015;2(1):61-73.

7 Betsch C, Böhm R, Korn L, Holtmann C. On the benefits of explaining herd immunity in vaccine advocacy. Nature human behaviour 2017;1(3):1-6.

8 Betsch C, Schmid P, Heinemeier D, Korn L, Holtmann C, Böhm R. Beyond confidence: Development of a measure assessing the $5 \mathrm{C}$ psychological antecedents of vaccination. PloS one 2018;13(12):e0208601.

9 Dubé E, Laberge C, Guay M, Bramadat P, Roy R, Bettinger JA. Vaccine hesitancy: an overview. Human vaccines \& immunotherapeutics 2013;9(8):1763-1773.

10 Fine P, Eames K, Heymann DL. "Herd immunity": a rough guide. Clinical infectious diseases 2011;52(7):911-916.

11 Freeman D, Haselton P, Freeman J, Spanlang B, Kishore S, Albery E, et al. Automated psychological therapy using immersive virtual reality for treatment of fear of heights: a singleblind, parallel-group, randomised controlled trial. The Lancet Psychiatry 2018;5(8):625-632.

12 Geiger M, Rees F, Lilleholt L, Santana AP, Zettler I, Wilhelm O, et al. Measuring the 7Cs of vaccination readiness. European Journal of Psychological Assessment 2021;

13 Haas EJ, Angulo FJ, McLaughlin JM, Anis E, Singer SR, Khan F, et al. Impact and effectiveness of mRNA BNT162b2 vaccine against SARS-CoV-2 infections and COVID-19 cases, hospitalisations, and deaths following a nationwide vaccination campaign in Israel: an observational study using national surveillance data. The Lancet 2021;397(10287):1819-1829.

14 Hakim H, Provencher T, Chambers CT, Driedger SM, Dube E, Gavaruzzi T, et al. Interventions to help people understand community immunity: a systematic review. Vaccine 2019;37(2):235247.

15 Hendrix KS, Finnell SME, Zimet GD, Sturm LA, Lane KA, Downs SM. Vaccine message framing and parents' intent to immunize their infants for MMR. Pediatrics 2014;134(3):e675e683.

16 MacDonald NE, et al. Vaccine hesitancy: Definition, scope and determinants. Vaccine 2015;33(34):4161-4164.

17 Makransky G, Andreasen NK, Baceviciute S, Mayer RE. Immersive virtual reality increases liking but not learning with a science simulation and generative learning strategies promote learning in immersive virtual reality. Journal of Educational Psychology 2020;.

18 Makransky G, Petersen GB. The Cognitive Affective Model of Immersive Learning (CAMIL): a Theoretical Research-Based Model of Learning in Immersive Virtual Reality. Educational Psychology Review 2021;p. 1-22. 
19 Nicholls LAB, Gallant AJ, Cogan N, Rasmussen S, Young D, Williams L. Older adults' vaccine hesitancy: psychosocial factors associated with influenza, pneumococcal, and shingles vaccine uptake. Vaccine 2021;39(26):3520-3527.

20 Nowak GJ, Evans NJ, Wojdynski BW, Ahn SJG, Len-Rios ME, Carera K, et al. Using immersive virtual reality to improve the beliefs and intentions of influenza vaccine avoidant 18-to-49-year-olds: considerations, effects, and lessons learned. Vaccine 2020;38(5):12251233.

21 Parong J, Mayer RE. Learning science in immersive virtual reality. Journal of Educational Psychology 2018;110(6):785.

22 Petersen GB, Klingenberg S, Mayer RE, Makransky G. The virtual field trip: Investigating how to optimize immersive virtual learning in climate change education. British Journal of Educational Technology 2020;51(6):2099-2115.

23 Pfattheicher S, Petersen MB, Böhm R. Information about herd immunity through vaccination and empathy promote COVID-19 vaccination intentions. Health Psychology 2021;Forthcoming.

24 Sanchez-Vives MV, Slater M. From presence to consciousness through virtual reality. Nature Reviews Neuroscience 2005;6(4):332-339.

25 Sprengholz P, Betsch C. Herd immunity communication counters detrimental effects of selective vaccination mandates: Experimental evidence. EClinicalMedicine 2020;22:100352.

$26 \mathrm{Wu} \mathrm{B}, \mathrm{Yu} \mathrm{X}, \mathrm{Gu} \mathrm{X}$. Effectiveness of immersive virtual reality using head-mounted displays on learning performance: A meta-analysis. British Journal of Educational Technology 2020;51(6):1991-2005. 